\title{
Distribution of Plasmodium species
} and assessment of performance of diagnostic tools used during a malaria survey in Southern and Western Provinces of Zambia

Lungowe Sitali ${ }^{1,2,3^{*}} \mathbb{C}$, John M. Miller ${ }^{4}$, Mulenga C. Mwenda ${ }^{4}$, Daniel J. Bridges ${ }^{4}$, Moonga B. Hawela ${ }^{5}$, Busiku Hamainza ${ }^{5}$, Elizabeth Chizema-Kawesha ${ }^{5}$, Thomas P. Eisele ${ }^{6,7}$, James Chipeta ${ }^{3,8}$ and Bernt Lindtjørn ${ }^{1}$

\begin{abstract}
Background: Zambia continues to make strides in reducing malaria burden through the use of proven malaria interventions and has recently pledged to eliminate malaria by 2021. Case management services have been scaled up at community level with rapid diagnostic tests (RDTs) providing antigen-based detection of falciparum malaria only. Key to national malaria elimination goals is the ability to identify, treat and eliminate all Plasmodium species. This study sought to determine the distribution of non-falciparum malaria and assess the performance of diagnostic tests for Plasmodium falciparum in Western and Southern Provinces of Zambia, two provinces planned for early malaria elimination.

Methods: A sub-set of individuals' data and samples from a cross-sectional household survey, conducted during peak malaria transmission season in April and May 2017, was used. The survey collected socio-demographic information on household members and coverage of malaria interventions. Malaria testing was done on respondents of all ages using blood smears and RDTs while dried blood spots were collected on filter papers for analysis using photoinduced electron transfer polymerase chain reaction (PET-PCR). Slides were stained using Giemsa stain and examined by microscopy for malaria parasites.
\end{abstract}

Results: From the 1567 individuals included, the overall prevalence of malaria was 19.4\% (Cl 17.5-21.4) by PCR, 19.3\% (CI 17.4-21.4) by RDT and 12.9\% (CI 11.3-14.7) by microscopy. Using PET-PCR as the gold standard, RDTs showed a sensitivity of $75.7 \%$ (Cl 70.4-80.4) and specificity of $94.2 \%$ (Cl 92.8-95.4). The positive predictive value (PPV) was 75.9\% (Cl 70.7-80.6) and negative predictive value (NPV) was 94.1\% (Cl 92.1-95.4). In contrast, microscopy for sensitivity, specificity, PPV, and NPV values were 56.9\% (Cl 51.1-62.5), 97.7\% (Cl 96.7-98.5), 85.6\% (Cl 80.0-90.2), 90.4\% (Cl 88.7-91.9), respectively. Non-falciparum infections were found only in Western Province, where $11.6 \%$ of $P$. falciparum infections were co-infections with Plasmodium ovale or Plasmodium malariae.

Conclusion: From the sub-set of survey data analysed, non-falciparum species are present and occurred as mixed infections. As expected, PET-PCR was slightly more sensitive than both malaria RDTs and microscopy to detecting malaria infections.

Keywords: Malaria, Mixed infections, Non-falciparum infection, Rapid diagnostic tests, Zambia

\footnotetext{
*Correspondence: lungowesitali@gmail.com

${ }^{1}$ Centre for International Health, Faculty of Medicine, University

of Bergen, Bergen, Norway

Full list of author information is available at the end of the article
} 


\section{Background}

Plasmodium falciparum is the major cause of malaria in Africa, while P. vivax is the most widely distributed species outside Africa [1], and in a few African countries, such as Ethiopia [2] and Uganda [3]. Compared with these two dominant species, Plasmodium malariae and Plasmodium ovale are significantly rarer and to a large extent are under-studied. Plasmodium ovale has been reported to be primarily distributed throughout sub-Saharan Africa [4]. Plasmodium malariae is found in tropical Africa where co-infections are sometimes encountered with $P$. falciparum [5].

Malaria remains a major public health problem in 91 countries worldwide, despite being preventable and treatable. It was linked to 216 million cases and 445,000 deaths in 2016, of which $90 \%$ were in sub-Saharan Africa [6]. Zambia has recorded a drop in malaria incidence from 407 cases per 1000 population in 2014 to 335 cases per 1000 population in 2015 [7] and it continues to make strides in reducing malaria cases through the use of proven and effective malaria interventions. It recently pledged to eliminate malaria altogether, through sustained universal coverage of vector control interventions, which include indoor residual spraying (IRS), distribution of long-lasting insecticide-treated nets (LLINs) and larval source management (LSM). Other important strategies include case management, health promotion, surveillance, and research [8]. Case management services are increasingly occurring at community level with standard rapid diagnostic tests (RDTs) providing antigenbased detection of falciparum malaria only and treatment with artemisinin-based combination therapy (ACT) for uncomplicated malaria coupled with injectable artesunate for severe cases. In addition, mass drug administration (MDA) has been included as an potential accelerator of the malaria elimination process [8].

Prompt and accurate case management of malaria infections is dependent on the performance of diagnostic tools. Readily available diagnostic tools include light microscopy of blood smears, RDTs, and molecular approaches such as polymerase chain reaction (PCR) and loop-mediated isothermal amplification (LAMP) $[9,10]$, although PCR is mostly used for research and not routine clinical diagnosis. Expert malaria microscopy remains an ideal diagnostic for malaria but due to a number of factors it cannot be used in all health facilities, hence the use of RDTs. RDTs are immunochromatographic tests that detect one or more of a range of antigens, namely histidine-rich protein 2 (HRP2), Plasmodium lactate dehydrogenase (pLDH) and aldolase [11]. Aldolase and pLDH are enzymes in the glycolytic pathway, while HRP2 is a water-soluble protein that is produced by asexual trophozoites and young gametocytes [12]. As HRP2 is produced exclusively during the asexual stages of the life cycle of $P$. falciparum, RDTs based on HRP2 detection are specific for P. falciparum [13], and are the only RDTs used in government health facilities in Zambia. RDTs are easy to use, do not require specialized training, can be performed in a clinic, health centre and hospital in the absence of electricity, and give results rapidly [14]. For these reasons, they have been widely adopted even at the lowest level in service delivery, the community level [15].

As with any diagnostic, there are limits to their utility and in some settings have shown poor sensitivity and specificity. For example, in a holo-endemic area of northern Tanzania, the sensitivity of the ParaHIT test was found to be $10.7 \%$ [16], while the sensitivity of the SD-Bioline test assessed in South Kivu Province of the Democratic Republic of the Congo was found to be $82.1 \%$ with a specificity of $92.0 \%$, using microscopy as the standard [17]. The World Health Organization (WHO) recommended a threshold for sensitivity of $>95 \%$ and a threshold for specificity of $>90$ [18].

As a country moves toward malaria elimination, it is crucial to ensure that all malaria cases irrespective of the infecting species are diagnosed and treated promptly. In Zambia, the distribution of Plasmodium species is not well defined. Despite this, HRP2-based RDTs are used for diagnosis in all facilities where microscopy is not available. This decision was based on information suggesting that $98 \%$ of malaria in Zambia was P. falciparum, $2 \%$ were $P$. malariae, while $P$. vivax is a rare infection [19], which may not have changed over the years. For example, a cross-sectional study conducted in high transmission areas in Eastern and Luapula Provinces revealed approximately $10.6 \%$ of all $P$. falciparum infections were coinfections with one or more other Plasmodium species. It is possible that in low transmission settings, the species distribution is different again, as species characterised by chronic infections ( $P$. malariae) or dormant lifecycle stages ( $P$. vivax and $P$. ovale) may constitute an increasing proportion of infections because of the chronic nature of $P$. malariae and the presence of the hypnozoite stages $P$. ovale and $P$. vivax [20]. These non-falciparum species potentially require additional interventions such as an anti-hypnozoite drug, e.g., primaquine.

\section{Methods}

Study design

A group of individuals were enrolled in across-sectional household survey conducted during peak malaria transmission season in April and May 2017, as part of ongoing efforts by the Zambia Ministry of Health, the PATH Malaria Control and Elimination Partnership in Africa (MACEPA) and other partners to evaluate malaria elimination efforts across Southern and Western Provinces. 
The survey collected socio-demographic information on household members, coverage of malaria interventions, and additional social and behavioural information related to use of malaria interventions. As well as testing for malaria in the field with an RDT (SD Bioline malaria Ag pf, Standard Diagnostics Inc., Republic of Korea), a thick blood smear and a dried blood spot (DBS) was collected for analysis at the National Malaria Elimination Centre (NMEC) laboratory.

The sampling methods for each province were different due to historical studies and enumeration in the two provinces. In Southern Province, there was a pre-existing sampling frame used during a previously implemented MDA trial. In the trial sampling from the 10 districts along Lake Kariba, 52 households were randomly selected from each of the 60 health facility catchment areas. In Western Province, where there was no pre-existing household sampling frame, a two-stage cluster sampling with clusters selected using probability proportional to size (a standardized method from the country's Malaria Indicator Survey) was used to select 25 households from 24 census-derived standard enumeration areas [21, 22]. All consenting or assenting individuals above 1 month of age $(n=6977)$ were enrolled in the two surveys. Those that were severely sick, were taken to clinic by survey staff, but information about them would be collected from the household respondent and no finger stick data would be collected.

With the help of OpenEpi software (Emory University, Rollins School of Public Health, USA) [23], 300 samples were calculated using the prevalence of different species at $10.6 \%$ [34] rounding up to $11 \%$ for high transmission areas, and estimating $2 \%$ in low transmission areas, accuracy could be determined with $80 \%$ power and $95 \%$ confidence.

After excluding 41 clusters that were outliers by RDT prevalence, a total of 13 clusters were then randomly selected: 6 from Western (high RDT prevalence) and 7 from Southern (low RDT prevalence). All individuals from these clusters with complete survey data, including RDT and microscopy results, together with an identifiable DBS were selected for PCR speciation analysis $(n=1567)$, while those with insufficient blood and missing data were excluded. Due to time and cost of doing PCRs, all 6977 samples could not be analysed.

\section{Study area}

The two provinces, Western and Southern, cover approximately $126,386 \mathrm{~km}^{2}$ (17\% Western) and $85,823 \mathrm{~km}^{2}(11 \%$ Southern) of the total Zambia landmass, and are home to 902,974 (Western) and 1,589,926 (Southern) people, according to the 2010 population census. The Zambezi River flows through both provinces and the plains cover about $10 \%$ of the total area of Western Province. Tongaspeaking people in Southern and Lozi-speaking people in Western are the predominant ethnic groups [24, 25]. A map of Zambia in Fig. 1 shows the location of these two provinces. Malaria transmission varies greatly across these two provinces, with traditionally higher transmission intensity in Southern Province along Lake Kariba and in areas of Western Province around the swamps and wetlands in Luampa, Kaoma and Nkeyema districts and along the Zambezi River basin [21, 22, 26]. The rest of the areas away from water bodies have low transmission.

\section{Laboratory methods \\ Microscopy}

Blood smears for microscopy were prepared in the field by trained biomedical scientists, air dried in a dust-free environment and stored in slide boxes. They were then transported to the NMEC where they were stained using $3 \%$ Giemsa for $45 \mathrm{~min}$. The slides were examined independently by two experienced biomedical scientists.

\section{DNA extraction}

DNA was extracted from $6 \mathrm{~mm}(\sim 13.8 \mu \mathrm{l}$ whole blood $)$ DBS punch(es)using a Qiagen DNA mini kit (Qiagen, Germany) and eluted in $100 \mu \mathrm{l}$. All RDT-positive samples were extracted alone, while RDT-negatives were extracted in pools of 10, and deconvoluted if positive.

\section{PCR analysis}

PET-PCR (real-time PCR technique), as previously described in 2013 by Lucchi et al. [27] was used to amplify Plasmodium 18S ribosomal RNA (see Table 1 materials for sequences). Briefly, $5 \mu$ of DNA template $(\sim 0.7 \mu \mathrm{l}$ whole blood) was amplified in a $20-\mu \mathrm{l}$ reaction as follows: $95{ }^{\circ} \mathrm{C}$ for $15 \mathrm{~min}$, followed by 45 cycles of $95{ }^{\circ} \mathrm{C}$ for $20 \mathrm{~s}$ and $60{ }^{\circ} \mathrm{C}$ for $40 \mathrm{~s}$. Samples were analysed in duplicate and scored positive if both duplicates had a CT value $<40$. The amplicon sizes were $109 \mathrm{bp} P$. falciparum, $137 \mathrm{bp} P$. malariae, $74 \mathrm{bp} P$. ovale, and $82 \mathrm{bp} P$. vivax [28, 29].

\section{Statistical analysis}

Demographic and laboratory data of participants' records were analysed using Stata version 13 (College Station, Texas, USA). Fisher's exact test for proportions was used to assess the association between variables and mixed and non-falciparum infection.

Diagnostic method performance was assessed against PCR as the gold standard as sensitivity [true positive $(\mathrm{TP}) /(\mathrm{TP}+$ false negative $(\mathrm{FN}))]$, specificity $[(\mathrm{TN}) /$ $(\mathrm{TN}+$ false positive $(\mathrm{FP}))]$, positive predictive value (PPV) $[\mathrm{TP} /(\mathrm{TP}+\mathrm{FP})]$ and negative predictive value $(\mathrm{NPV})[\mathrm{TN} /(\mathrm{TN}+\mathrm{FN})]$. The results were interpreted 

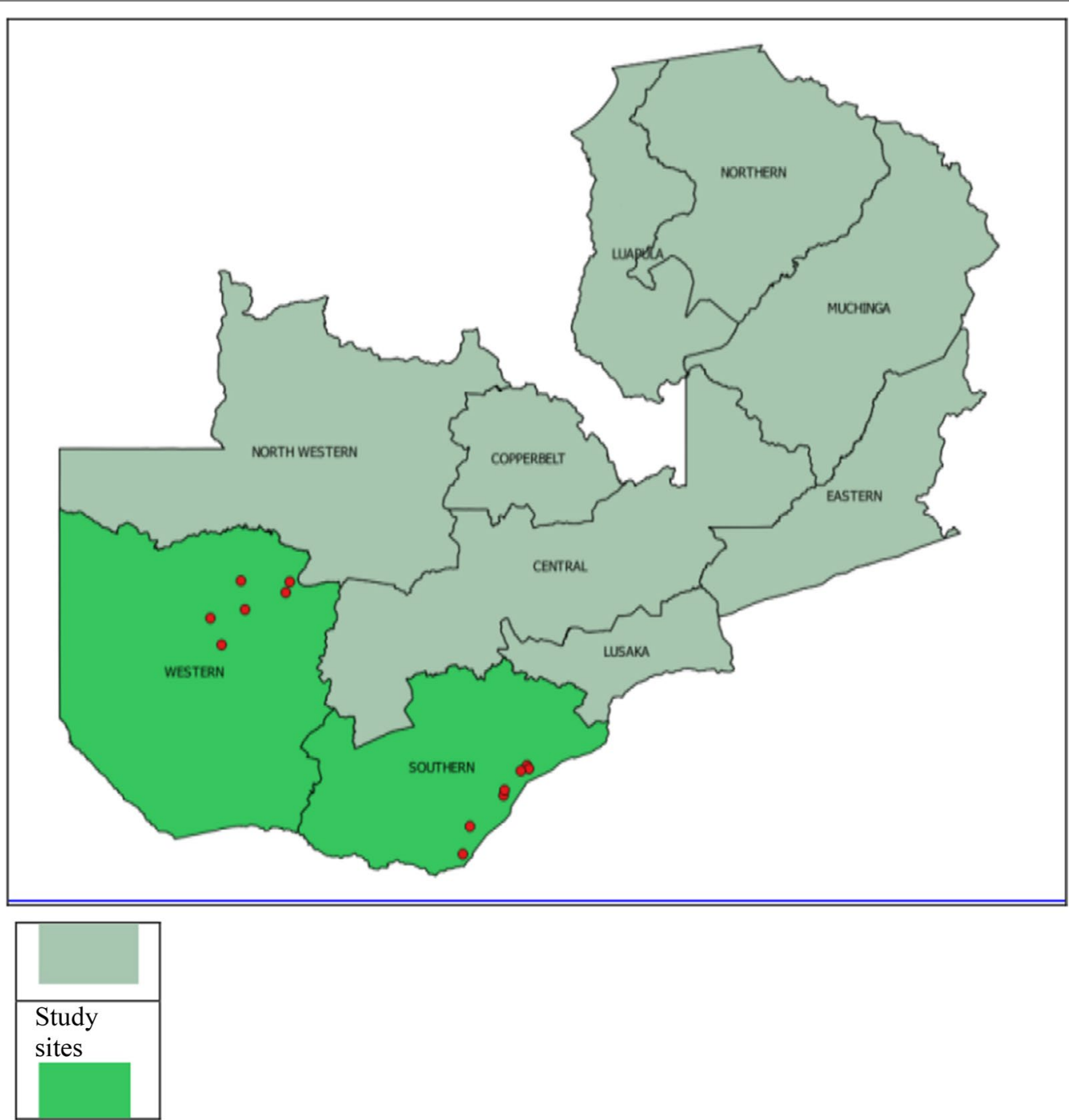

Fig. 1 Map of Zambia showing location of Southern and Western Provinces. The study area is highlighted showing the red circles indicate the exact locations where samples were collected in the two provinces

Table 1 Primers for species identification used for PET-PCR

\begin{tabular}{|c|c|}
\hline Primer name & Sequence $\left(5^{\prime}-3^{\prime}\right)$ \\
\hline Original genus 18sFor & GGC CTA ACA TGG CTA TGA CG \\
\hline Original genus FAM 18sRev & FAM-aggcgcatagcgcctggCTGCCTTCCTTAG ATGTGG TAG CT \\
\hline Falciparum For & ACC CCTCGCCTG GTG TTTTT \\
\hline Falciparum Rev & HEX-aggcggataccgcctggTCGG GCC CCA AAA ATA GGA A \\
\hline P. vivax For & GTA GCC TAAGAAGGC CGT GT \\
\hline P. vivax Rev & HEX-aggcgcatagcgectggCCTGGGG GAT GAA TAT CTC TAC AGC ACT GT \\
\hline P. malariae For & AAGGCAGTAACACCAGCAGTA \\
\hline $\begin{array}{l}\text { P. malariae Rev (based on dihydrofolate reductase-thymidylate synthase } \\
\text { (DHFR-TS) gene) }\end{array}$ & FAM-aggcgcatagcgcctggTCCCATGAAGTTATATTCCCGCTC \\
\hline P. ovale For & FAM-aggcgcatagcgcctggCCACAGATAAGAAGTCTCAAGTACGATATT \\
\hline P. ovale Rev & TTGGAGCACTTTTGTTTGCAA \\
\hline
\end{tabular}

Table showing forward and reverse primers for species identification used in PET-PCT assay 
with 95\% confidence intervals (CIs) GraphPad prism (GraphPad software Inc, San Diego, USA) was used to calculate Cohen's Kappa agreement coefficient.

\section{Results}

Table 2 shows the general and socio-demographic characteristics of the individuals included in this study. Age, gender, reported travel history, LLIN ownership were similar across both provinces, while IRS coverage was markedly higher in Southern (67.2\%) compared to Western Province (31.6\%).

Malaria prevalence determined by RDT or PCR was broadly similar across both provinces, while microscopy was significantly lower. In contrast, Western Province had a significantly higher prevalence of malaria as compared to Southern, 55 versus $4 \%$ by PCR (Fig. 2). The majority of all PCR-positive infections were P. falciparum mono-infections with 85.7 and $97.8 \%$ in Western and Southern, respectively. No mixed/co-infections were found in Southern Province, but $11.6 \%$ of all positives in Western had more than one species present (Fig. 3).

Table 2 General and socio-demographic characteristics of participants

\begin{tabular}{|c|c|c|c|c|}
\hline \multirow[t]{2}{*}{ Characteristics } & \multicolumn{2}{|c|}{ Southern; n= 1096} & \multicolumn{2}{|c|}{ Western; $n=471$} \\
\hline & n (\%) & $\mathrm{Cl}$ & n (\%) & $\mathrm{Cl}$ \\
\hline \multicolumn{5}{|l|}{ Gender } \\
\hline Male & $505(46.1)$ & $43.6-48.5$ & $210(44.6)$ & $39.5-49.8$ \\
\hline Female & $591(53.9)$ & $51.5-56.4$ & $261(55.4)$ & $50.2-60.5$ \\
\hline \multicolumn{5}{|l|}{ Age of children (years) } \\
\hline$<5$ & $197(18.0)$ & $14.2-22.5$ & $86(18.3)$ & $15.6-21.2$ \\
\hline $5-10$ & $200(18.3)$ & $16.0-20.7$ & $87(18.5)$ & $14.9-22.6$ \\
\hline $11-15$ & $178(16.2)$ & $13.5-19.4$ & $69(14.7)$ & 10.6-20.0 \\
\hline $16-25$ & $140(12.8)$ & $10.5-15.5$ & $72(15.3)$ & $11.8-19.6$ \\
\hline $26-40$ & $191(17.4)$ & $15.1-20.0$ & $59(12.5)$ & $7.4-20.3$ \\
\hline $40-94$ & $190(17.7)$ & $14.3-20.8$ & $98(20.8)$ & $17.6-24.4$ \\
\hline \multicolumn{5}{|l|}{ Travel history } \\
\hline Yes & $6(0.5)$ & $0.2-1.4$ & $23(4.9)$ & $1.7-13.2$ \\
\hline No & 1089 (99.5) & $98.6-99.8$ & $445(95.1)$ & $86.8-98.3$ \\
\hline \multicolumn{5}{|l|}{ Household sprayed } \\
\hline Yes & $730(67.2)$ & $52.4-79.1$ & $149(31.6)$ & $8.7-69.1$ \\
\hline No & $357(32.8)$ & $20.8-47.6$ & $322(68.4)$ & $30.9-91.2$ \\
\hline \multicolumn{5}{|l|}{ ITN ownership } \\
\hline Yes & $673(61.4)$ & $38.8-80.0$ & $269(57.1)$ & $29.2-81.1$ \\
\hline No & $13(1.2)$ & $0.1-9.7$ & $8(1.7)$ & $0.3-8.6$ \\
\hline Missing information & $410(37.4)$ & $18.7-60.8$ & $194(41.2)$ & $17.1-70.4$ \\
\hline \multicolumn{5}{|l|}{ Cluster } \\
\hline $\begin{array}{l}\text { Average no of people/ } \\
\text { cluster }\end{array}$ & 156.5 & & 78.5 & \\
\hline Number of clusters & 7 & & 6 & \\
\hline
\end{tabular}

The tables shows the general and socio-demographic characteristics of the participant in the two study areas

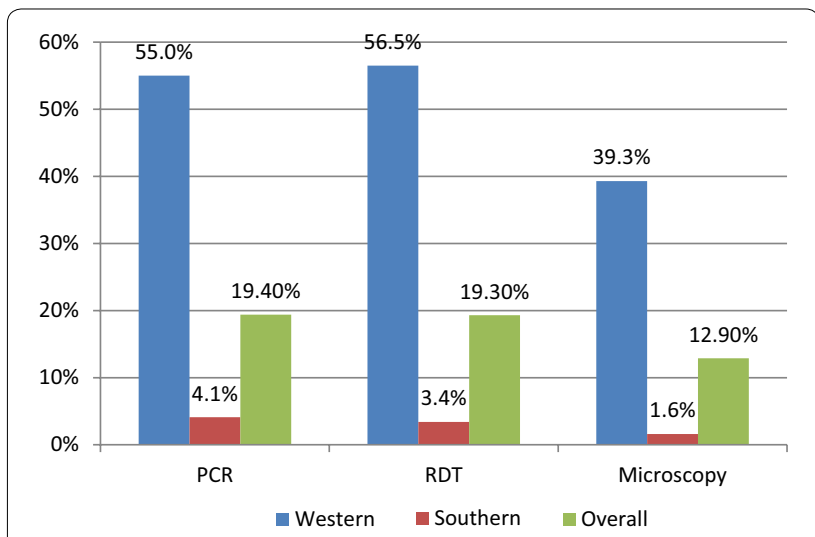

Fig. 2 Prevalence of malaria among the participants by province

A total of 8 non-falciparum mono-infections were also identified. Of these, 6 were $P$. ovale infections (exclusively from Western Province) and 2 P. malariae infections, one in each Province (Table 3).

\section{Sensitivity, specificity, positive, and negative predictive} values of diagnostic tools

RDTs had sensitivity of 75.5\% [230/304 (95\% CI 70.4$80.4 \%)]$ and a specificity of $94.2 \%$ [1190/1263 (95\% CI 92.8-95.4\%)], with a PPV of $75.9 \%$ [230/303 (95\% CI 70.7-80.6\%)] and NPV of 1190/1264 [94.1\% (95\% CI 92.1-95.4\%)]. The observed agreement percentage was 90.97\%, and Cohen's Kappa was 0.71 (CI 0.66-0.75). Microscopy was observed to have a sensitivity of $56.9 \%$ [173/304 (95\% CI 51.1-62.5)], specificity of $97.7 \%$ [1234/1264 (95\% CI 96.7-98.5)], PPV of 85.6\% [173/202 (95\% CI 80.0-90.2)] and an NPV of 90.4\% [1234/1365 (95\% CI 88.7-91.9)]. The observed agreement percentage was $89.79 \%$ and a Cohen's Kappa of 0.63 (CI 0.57-0.68) (Table 4). Results from RDTs and microscopy against PCR showed a substantial measure of agreement.

Table 5 shows the sensitivity and specificity for RDTs when the standard is microscopy. The sensitivity was $81.2 \%$ [164/202 (95\% CI 75.1-86.3\%)] and the specificity was $89.8 \%$ [1226/1365 (95\% CI 88.1-91.4)], with a PPV of $54.1 \%$ [164/303 (95\% CI 48.3-59.8)] and an NPV of 97.0\% [1226/1264 (95\% CI 95.9-97.8)]. The observed agreement percentage was $88.70 \%$ and a Cohen's Kappa of 0.0 .59 (CI 0.53-0.64). A moderate measure of agreement was observed. Cohen's Kappa was calculated using graph pad online calculate [30].

\section{Discussion}

This study identified the presence of four Plasmodium species (P. falciparum, $P$. ovale, $P$. malariae, $P$. vivax) in Western Province and two (P. falciparum, P. malariae) 


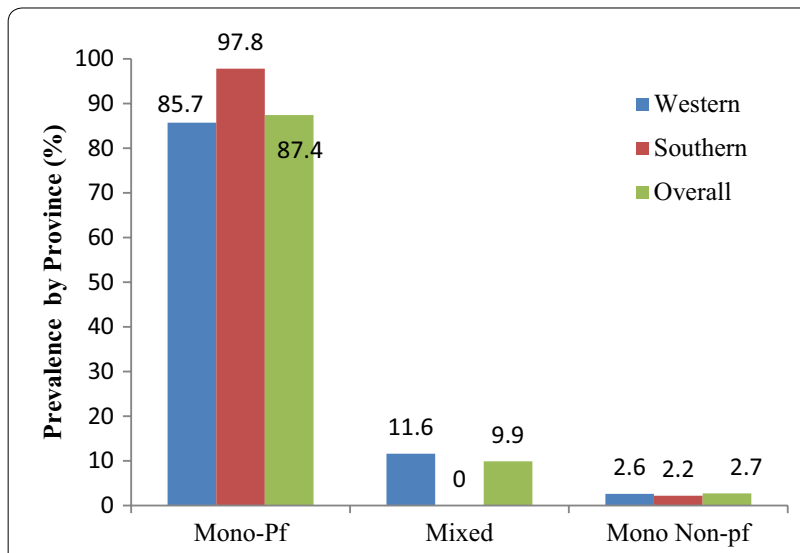

Fig. 3 Prevalence of Plasmodium falciparum, mixed infection and mono non-falciparum infection by PET-PCR in Southern Province. Interestingly, the diversity of parasite species found in a province broadly correlated to the malaria prevalence, i.e., the higher the prevalence the greater the number of species found. From these data alone, it seems clear that non-falciparum infections are under-reported due to the use of $P$. falciparum-specific RDTs and challenges in achieving high quality microscopy [31, 32].

Overall, 97\% of all malaria infections contained $P$. falciparum, of which $89 \%$ were mono-infections and $11.6 \%$ co-infections and very few mono non-falciparum infections were identified ( $<3 \%$ of all infections). These findings are in agreement with previous studies which reported $10.6 \%$ mixed infections and $88 \%$ P. falciparum [33], and are also close to $98 \%$ P. falciparum reported by Wolfe [34]. The findings suggest that transmission is occurring through a common vector population or

Table 3 Differential species distribution by province

\begin{tabular}{|c|c|c|c|c|c|c|}
\hline \multirow[t]{2}{*}{ Province } & \multicolumn{2}{|c|}{ Western } & \multicolumn{2}{|c|}{ Southern } & \multicolumn{2}{|c|}{ Overall } \\
\hline & $\mathrm{n}$ & $\%$ & $\mathrm{n}$ & $\%$ & $\mathrm{n}$ & $\%$ \\
\hline Pf only & 222 & 85.7 & 44 & 97.8 & 266 & 87.5 \\
\hline Po only & 6 & 2.3 & & & 6 & 2.0 \\
\hline Pm only & 1 & 0.4 & 1 & 2.2 & 2 & 0.7 \\
\hline Pf and Pv & 1 & 0.4 & & & 1 & 0.3 \\
\hline Pf and Po & 26 & 10.0 & & & 26 & 8.6 \\
\hline Pf and Pm & 3 & 1.2 & & & 3 & 1.0 \\
\hline Total & 259 & & 45 & & 304 & \\
\hline
\end{tabular}

The majority of the infections were P. falciparum, with (222/259) $85.7 \%$ and (44/45) $97.8 \%$ in Western and Southern, respectively. There were (30/259) $11.6 \%(95 \% \mathrm{Cl}$ 8.4-16.0\%) mixed infections in Western, while none were observed in Southern Provinces. The combination observed in Western Province were Pf/Pv 1/259 (0.4\%), Pf/ Po $26 / 259$ (10.0\%). Pf/Pm 3/259 (1.2\%)

Pf: Plasmodium falciparum; Pm: P. malariae; Po: P. ovale; Pv: P. vivax

Table 4 Performance of RDTs and microscopy compared to PCR results

\begin{tabular}{|c|c|c|c|c|c|c|c|}
\hline & \multicolumn{2}{|l|}{ PCR } & & \multirow[t]{2}{*}{ Sensitivity (95\% CI) } & \multirow[t]{2}{*}{ Specificity (95\% Cl) } & \multirow[t]{2}{*}{ PPV $(95 \% \mathrm{Cl})$} & \multirow[t]{2}{*}{ NPV $(95 \% \mathrm{Cl})$} \\
\hline & Positive & Negative & & & & & \\
\hline \multicolumn{8}{|l|}{ RDTs } \\
\hline Positive & 230 & 73 & 303 & $75.5 \%(70.4,80.4)$ & $94.2 \%(92.8,95.4)$ & $75.9 \%(70.7,80.6)$ & $94.1 \%(92.1,95.4)$ \\
\hline Negative & 74 & 1190 & 1264 & & & & \\
\hline \multirow[t]{3}{*}{ Total } & 304 & 1263 & 1567 & & & & \\
\hline & \multicolumn{2}{|l|}{ PCR } & & Sensitivity $(95 \% \mathrm{CI})$ & Specificity (95\% Cl) & PPV $(95 \%$ Cl) & NPV (95\% Cl) \\
\hline & Positive & Negative & & & & & \\
\hline \multicolumn{8}{|l|}{ Microscopy } \\
\hline Positive & 173 & 29 & 202 & $56.9 \%(51.1,62.5)$ & $97.7 \%(96.7,98.5)$ & $85.6 \%(80.0,90.2)$ & $90.4 \%(88.7,91.9)$ \\
\hline Negative & 131 & 1234 & 1365 & & & & \\
\hline Total & 304 & 1264 & 1567 & & & & \\
\hline
\end{tabular}

PPN: positive predictive value; NPV: negative predictive value 
Table 5 Performance of RDTs compared with microscopy results

\begin{tabular}{lcccc}
\hline RDT & \multicolumn{2}{l}{ Microscopy } & \\
\cline { 2 - 4 } & Positive & Negative & Total & \\
\hline Positive & 164 & 139 & 303 & $\mathrm{Se}=81.2 \%(75.1,86.3)$, \\
Negative & 38 & 1226 & 1264 & $\mathrm{Sp}=89.8 \%(88.1,91.4)$, \\
Total & 202 & 1365 & 1567 & $\begin{array}{l}\text { Ppv }=54.1 \%(48.3, \\
\end{array}$ \\
& & & & $(95.8), \mathrm{Npv}=97.0 \%$ \\
& & & & \\
\hline
\end{tabular}

that transmission of each species is occurring in the same geography/human population. It is well known that some mosquito species are capable of transmitting multiple parasite species, e.g. Anopheles gambiae is able to transmit all Plasmodium species [35] and is found throughout Southern and Western Provinces. While the vectorial capacity for transmission is present in both provinces, it is unclear how much non-P. falciparum transmission is local and how much is imported through travel [35]. Considering the numbers, it is entirely plausible that $P$. malariae in Southern Province is maintained through importation, while there is more robust local non-P. falciparum transmission in Western Province. No association was found between travel history and presence of non-falciparum infections however, only travel in the last month was recorded. Considering non-falciparum malaria infections may be chronic or dormant for long periods of time, it is not possible to determine the source of infections in this study. Genotyping of the parasite population may help dissect this relationship by defining the parasite population diversity and relatedness of different infections.

Often the non-falciparum species are under-reported or not identified due to a number of factors: the use of $P$. falciparum-specific RDTs as observed in approximately $63 \%$ of health facilities in Zambia [36] and microscopyrelated challenges, such as inadequate experience and training of microscopists to identify parasites other than P. falciparum [31, 32]. Other factors include morphological changes induced by haemolysis hampering the identification of the species [31].

While evidence for P. vivax transmission exists [37], the majority of the Zambian population are expected to be resistant to $P$. vivax infections due to carrying the Duffy FyFy genotype [38]. It was, therefore, surprising to find $P$. vivax, albeit in only one person. There is evidence of low-level $P$. vivax endemicity in sub-Saharan Africa [39, 40]. Evidence from the Malaria Atlas Project (MAP) shows that Zambia's neighbours, Democratic Republic of Congo, Namibia, Botswana and Angola, have cases of P. vivax [39].
The sensitivity value was below the 95\% threshold for both RDTs and microscopy when compared to PCR, while specificity was above the recommended $90 \%$. The findings of RDT sensitivity of $75.5 \%$ using PCR as gold standard is consistent with other publications on RDT sensitivity e.g., 88.6\% in mainland Tanzania [41], 76.5\% in Zanzibar [42] and 75.4\% in Kisumu, Kenya [43].

In this study RDTs and microscopy had a relatively low PPV $(<90 \%)$, meaning that many positives were not infected, which in turn affects malaria morbidity, prevalence and incidence estimates [43]. Furthermore, treatment given to false positives is potentially costly and may lead to inaccurate perceptions of therapeutic failures [43]. A proportion of false positive individuals may reflect a recent resolved past infection due to HRP2 persistence. Conversely, a proportion of false negatives could be due to the functional loss of HRP2 expression, e.g., through a gene deletion as has been reported in neighbouring DRC [44]. Finally, the prozone effect in hyperparasitaemic infections could also account for some of the false negatives $[45,46]$, although in this study the correlation between parasite density and false negatives was not assessed.

\section{Study limitations and strengths}

Results of this study should be interpreted keeping some limitations in mind. Firstly, there was no similar pre2017 analysis of species in Southern Province to indicate whether there was previously a higher level of mixed and non-falciparum species present. This makes it difficult to be certain if the elimination activities in the province are responsible for the low positivity rate. Secondly, these data cannot be generalized to the whole country, as this analysis is for two provinces, despite representing lower and higher transmission zones (low meaning an area with parasitaemia less than 5\% and high meaning an area with parasitaemia above 15\%), and the moderate transmission zone is not represented. Thirdly, an element of selection bias cannot be excluded as clusters with zero RDT prevalence were excluded. It is possible that the clusters may have had non-falciparum species leading to underestimation of the species. Finally, the study used secondary data and the authors have little or no control of RDT results, which may have affected the sensitivity and specificity results. Four samples from participants that were RDTpositive and PCR-negative, who were given treatment within 14 days, were included in the analysis. It is possible that the positive RDTs could have been due to lagging antigenaemia. Although on recalculation of sensitivity and specificity, new figures were within the same range as those previously calculated. 


\section{Conclusion}

There is a concern that other species could continue to drive transmission but remain undetected, as the RDTs currently used in Zambia detect only P. falciparum. While this study confirms that $P$. falciparum dominates, a not-insignificant $9.9 \%$ of these infections were mixed with another species. Encouragingly, where malaria is closest to elimination (i.e., in Southern Province), nonfalciparum infections identified in this study were minimal (1 case). This may suggest that interventions that reduce $P$. falciparum transmission also impact non-falciparum species. It is important to expand surveillance activities to monitor non-falciparum infections and ensure this remains the case.

From the finding, recommendation made include: the national malaria programme consider increasing capacity to diagnose and detect non-falciparum species. This can be done through strengthening diagnosis quality assurance, increase access to functional microscopy, and provide refresher training in malaria microscopy for all laboratory staff, and in addition, introduce Pan RDTs (RDTs able to diagnose $P$. falciparum and non-falciparum species).

\begin{abstract}
Abbreviations
AL: artemether-lumefantrine; HRP2: histidine-rich protein 2; DBS: dried blood spot; DNA: deoxyribonucleic acid; ITN: insecticide-treated net; IRS: indoor residual spraying; LLINs: long-lasting insecticide-treated nets; LSM: larval source management; NPV: negative predictive value; NMEC: National Malaria Elimination Centre; NMCP: National Malaria Control Programme; MACEPA: Malaria Control and Elimination Partnership in Africa; MDA: mass drug administration; MIS: Malaria Indicator Survey; PCR: polymerase chain reaction; PET-PCR: photo-induced electronic transfer-polymerase chain reaction; $\mathrm{PLDH}$ : parasite lactate dehydrogenase; PPV: positive predictive value; RDTs: rapid diagnostic tests.
\end{abstract}

\section{Authors' contributions}

LS conceived the idea, run the samples and drafted the manuscript, MCM assisted in the running of the samples and writing the methods and correcting the manuscript. JM, TPE helped design and undertake of the original study and in the extraction and analysis of the data, and also contributed to the writing and correction of the manuscript. $\mathrm{MBH}, \mathrm{DJB}, \mathrm{BH}$ and ECK facilitated the running of the samples at the NMEC laboratory and helped in the writing and correction of the manuscript. BL, JC contributed to the design of the study and manuscript correction. All authors read and approved the final manuscript.

\footnotetext{
Author details

${ }^{1}$ Centre for International Health, Faculty of Medicine, University of Bergen, Bergen, Norway. ${ }^{2}$ Department of Biomedical Science, School of Health Sciences, University of Zambia, Lusaka, Zambia. ${ }^{3}$ School of Medicine and University Teaching Hospital Malaria Research Unit (SMUTH-MRU), Lusaka, Zambia. ${ }^{4}$ PATH Malaria Control and Elimination Partnership in Africa (MACEPA), National Malaria Elimination Centre, Ministry of Health, Chainama Grounds, Lusaka, Zambia. ${ }^{5}$ National Malaria Elimination Centre, Ministry of Health, Chainama Hospital and College Grounds, Lusaka, Zambia. ${ }^{6}$ Department of Tropical Medicine, Tulane University School of Public Health and Tropical Medicine, New Orleans, LA, USA. ${ }^{7}$ Center for Applied Malaria Research and Evaluation, Tulane University School of Public Health and Tropical Medicine, New Orleans, LA, USA. ${ }^{8}$ Department of Paediatrics and Child Health, University of Zambia School of Medicine, Lusaka, Zambia.
}

\section{Acknowledgements}

We acknowledge role played by PATH Malaria Control and Elimination Partnership in Africa (MACEPA) in providing reagents and the Ministry of Health through the National Malaria Elimination Centre (NMEC) for allowing us to use the samples, the NMEC molecular laboratory team, Conceptor Mulube, Sandra Chishimba, Brenda Mambwe for the assistance rendered during the running of the samples. We also wish to thank Maya Fraser and Travis Porter from PATH-MACEPA for helping with data extraction process; and we wish to thank Manny Lewis from PATH-MACEPA for proof reading the manuscript.

\section{Competing interests}

The authors declare that they have no competing interests.

\section{Availability of data and materials}

The datasets generated and/or analysed during the current study are available from the corresponding author on reasonable request.

\section{Consent for publication}

Not applicable.

\section{Ethics approval and consent to participate}

Ethical clearance was obtained from the Regional Committee for Medical and Health Research Ethics (REC Western Norway) Ref no. 2016/1393/REK Vest and from the University of Zambia Biomedical Research Ethics Committee (UNZABREC) Ref no. 010-05-16. As this analysis was part of a larger study, ethical clearance for the larger study was also obtained from UNZABREC. Permission to use data was obtained from the Ministry of Health. All data analysed were anonymized.

\section{Funding}

This work was funded by Norwegian Loan Scheme Lånakassen and the Ministry of Health through MACEPA.

\section{Publisher's Note}

Springer Nature remains neutral with regard to jurisdictional claims in published maps and institutional affiliations.

Received: 30 November 2018 Accepted: 4 April 2019

Published online: 11 April 2019

\section{References}

1. Gething PW, Elyazar IR, Moyes CL, Smith DL, Battle KE, Guerra CA, et al. A long neglected world malaria map: Plasmodium vivax endemicity in 2010. PLoS Negl Trop Dis. 2012;6:e1814.

2. Geleta G, Ketema T. Severe malaria associated with Plasmodium falciparum and P. vivax among children in Pawe Hospital, Northwest Ethiopia. Malar Res Treat. 2016;2016:1240962.

3. Asua V, Tukwasibwe S, Conrad M, Walakira A, Nankabirwa JI, Mugenyi L, et al. Plasmodium species infecting children presenting with malaria in Uganda. Am J Trop Med Hyg. 2017;97:753-7.

4. Collins WE, Jeffery GM. Plasmodium ovale: parasite and disease. Clin Microbiol Rev. 2005; 18:570-81.

5. Autino B, Noris A, Russo R, Castelli F. Epidemiology of malaria in endemic areas. Mediterr J Hematol Infect Dis. 2012;4:e2012060.

6. WHO. World malaria report 2017. Geneva: World Health Organization; 2017.

7. Inambao AB, Kumar R, Hamainza B, Makasa M, Nielsen CF. Malaria incidence in Zambia, 2013 to 2015: observations from the health management information system. Health Press Zambia Bull. 2017;1(3):11-21.

8. Ministry of Health. National malaria elimination strategic plan 2017-2021. Lusaka: National Malaria Elimination Centre; 2017.

9. Bhandari PL, Raghuveer CV, Rajeev A, Bhandari PD. Comparative study of peripheral blood smear, quantitative buffy coat and modified centrifuged blood smear in malaria diagnosis. Indian J Pathol Microbiol. 2008; 1:108-12.

10. Khan MM, Kareem MA, Rao GK. Laboratory diagnosis of malaria infection and its natural history in an urban pocket of Hyderabad City. Indian J Malariol. 1989;26:215-8 
11. Moody AH, Chiodini PL. Non-microscopic method for malaria diagnosis using OptiMAL IT, a second-generation dipstick for malaria pLDH antigen detection. Br J Biomed Sci. 2002;59:228-31.

12. Murray CK, Bell D, Gasser RA, Wongsrichanalai C. Rapid diagnostic testing for malaria. Trop Med Int Health. 2003;8:876-83.

13. Howard RJ, Uni S, Aikawa M, Aley SB, Leech JH, Lew AM, et al. Secretion of a malarial histidine-rich protein (Pf HRP II) from Plasmodium falciparuminfected erythrocytes. J Cell Biol. 1986;103:1269-77.

14. Shiff CJ, Premji Z, Minjas JN. The rapid manual ParaSight-F test. A new diagnostic tool for Plasmodium falciparum infection. Trans $R$ Soc Trop Med Hyg. 1993;87:646-8.

15. Counihan H, Harvey SA, Sekeseke-Chinyama M, Hamainza B, Banda R, Malambo T, et al. Community health workers use malaria rapid diagnostic tests (RDTs) safely and accurately: results of a longitudinal study in Zambia. Am J Trop Med Hyg. 2012;87:57-63.

16. Kweka EJ, Lowassa A, Msangi S, Kimaro EE, Lyatuu EE, Mwang'onde $B J$, et al. Low sensitivity of ParaHIT-f rapid malaria test among patients with fever in rural health centers, Northern Tanzania. J Infect Dev Ctries. 2011;5:204-8.

17. Kashosi TM, Mutuga JM, Byadunia DS, Mutendela JK, Mulenda B, Mubagwa K. Performance of SD Bioline Malaria Ag Pf/Pan rapid test in the diagnosis of malaria in South-Kivu, DR Congo. Pan Afr Med J. 2017;27:216.

18. Mouatcho JC, Goldring JP. Malaria rapid diagnostic tests: challenges and prospects. J Med Microbiol. 2013;62:1491-505.

19. Ministry of Health. Guidelines of diagnosis and treatment of malaria in Zambia. 4th ed. Lusaka: National Malaria Control Centre; 2014

20. Marques PX, Saute F, Pinto WV, Cardoso S, Pinto J, Alonso PL, et al. Plasmodium species mixed infections in two areas of Manhica district, Mozambique. Int J Biol Sci. 2005;1:96-102.

21. Ministry of Health. Malaria indicator survey 2015 report. Lusaka: Ministry of Health; 2015

22. Eisele TP, Silumbe K, Finn T, Chalwe V, Kamuliwo M, Hamainza B, et al. Assessing the effectiveness of household-level focal mass drug administration and community-wide mass drug administration for reducing malaria parasite infection prevalence and incidence in Southern Province, Zambia: study protocol for a community randomized controlled trial. Trials. 2015;16:347.

23. Open Epi. Open Source epidemiologic statistics. https://www.opene pi.com/Menu/OE_Menu.htm.

24. CSO. 2010 census of population and housing; Southern province analytical report. Lusaka: CSO; 2010. p. 2014.

25. CSO. 2010 census of population and house; Western province analytical report. Lusaka: CSO; 2010. p. 2014.

26. Eisele TP, Bennett A, Silumbe K, Finn TP, Chalwe V, Kamuliwo M, et al. Short-term impact of mass drug administration with dihydroartemisinin plus piperaquine on malaria in Southern Province Zambia: a clusterrandomized controlled trial. J Infect Dis. 2016;214:1831-9.

27. Lucchi NW, Narayanan J, Karell MA, Xayavong M, Kariuki S, DaSilva AJ, et al. Molecular diagnosis of malaria by photo-induced electron transfer fluorogenic primers: PET-PCR. PLOS ONE. 2013;8:e56677.

28. Akerele D, Ljolje D, Talundzic E, Udhayakumar V, Lucchi NW. Molecular diagnosis of Plasmodium ovale by photo-induced electron transfer fluorogenic primers: PET-PCR. PLOS ONE. 2017;12:e0179178.
29. Lucchi NW, Karell MA, Journel I, Rogier E, Goldman I, Ljolje D, et al. PETPCR method for the molecular detection of malaria parasites in a national malaria surveillance study in Haiti, 2011. Malar J. 2014;13:462.

30. Motulsky H. GraphPad. 1989.

31. Mohapatra PK, Prakash A, Bhattacharyya DR, Goswami BK, Ahmed A, Sarmah B, et al. Detection \& molecular confirmation of a focus of Plasmodium malariae in Arunachal Pradesh, India. Indian J Med Res. 2008;128:52-6.

32. Cao YY, Wang WM, Zhou HY, Zhu GD, Xu S, Gu YP, et al. Cases diagnosis of imported malaria in Jiangsu province, 2014-2016. Zhonghua Liu Xing Bing Xue Za Zhi. 2018;39:218-21 (in Chinese)

33. Sitali L, Chipeta J, Miller JM, Moonga HB, Kumar N, Moss WJ, et al. Patterns of mixed Plasmodium species infections among children six years and under in selected malaria hyper-endemic communities of Zambia: population-based survey observations. BMC Infect Dis. 2015;15:204.

34. Wolfe HL. Plasmodium ovale in Zambia. Bull World Health Organ. 1968;39:947-8.

35. Mayxay M, Pukrittayakamee S, Newton PN, White NJ. Mixed-species malaria infections in humans. Trends Parasitol. 2004;20:233-40.

36. Hamer DH, Ndhlovu M, Zurovac D, Fox M, Yeboah-Antwi K, Chanda P, et al. Improved diagnostic testing and malaria treatment practices in Zambia. JAMA. 2007;297:2227-31.

37. Blossom DB, King CH, Armitage KB. Occult Plasmodium vivax infection diagnosed by a polymerase chain reaction-based detection system: a case report. Am J Trop Med Hyg. 2005;73:188-90.

38. Miller HL, Mason SJ, Clyde DF, McGinniss HM. The resistance factor to Plasmodium vivax in blacks. The Duffy-blood-group genotype, FyFy. N Engl J Med. 1976;295:302-4.

39. Howes RE, Reiner RC Jr, Battle KE, Longbottom J, Mappin B, Ordanovich $D$, et al. Plasmodium vivax transmission in Africa. PLoS Negl Trop Dis. 2015;9:e0004222.

40. Ryan JR, Stoute JA, Amon J, Dunton RF, Mtalib R, Koros J, et al. Evidence for transmission of Plasmodium vivax among a duffy antigen negative population in Western Kenya. Am J Trop Med Hyg. 2006;75:575-81.

41. Mahende C, Ngasala B, Lusingu J, Yong TS, Lushino P, Lemnge M, et al. Performance of rapid diagnostic test, blood-film microscopy and PCR for the diagnosis of malaria infection among febrile children from Korogwe District, Tanzania. Malar J. 2016;15:391.

42. Shakely D, Elfving K, Aydin-Schmidt B, Msellem MI, Morris U, Omar R, et al. The usefulness of rapid diagnostic tests in the new context of low malaria transmission in Zanzibar. PLoS ONE. 2013;8:e72912.

43. Wanja EW, Kuya N, Moranga C, Hickman M, Johnson JD, Moseti C, et al. Field evaluation of diagnostic performance of malaria rapid diagnostic tests in western Kenya. Malar J. 2016;15:456.

44. Doctor SM, Liu Y, Whitesell A, Thwai KL, Taylor SM, Janko M, et al. Malaria surveillance in the Democratic Republic of the Congo: comparison of microscopy, PCR, and rapid diagnostic test. Diagn Microbiol Infect Dis. 2016;85:16-8.

45. Gillet P, Mori M, Van EM, Van den Ende J, Jacobs J. Assessment of the prozone effect in malaria rapid diagnostic tests. Malar J. 2009;8:271.

46. Santos L, Pereira NR, Andrade P, Dias PF, Alves CL, Abreu C, et al. Prozonelike phenomenon in travellers with fatal malaria: report of two cases. J Infect Dev Ctries. 2015:9:321-4.

Ready to submit your research? Choose BMC and benefit from

- fast, convenient online submission

- thorough peer review by experienced researchers in your field

- rapid publication on acceptance

- support for research data, including large and complex data types

- gold Open Access which fosters wider collaboration and increased citations

- maximum visibility for your research: over 100M website views per year

At BMC, research is always in progress.

Learn more biomedcentral.com/submissions 